\title{
QoS-driven Channel Selection for Heterogeneous Cognitive Radio Networks
}

\author{
Luca Zappaterra \\ George Washington University \\ Washington, D.C. \\ lucaz@gwu.edu
}

\begin{abstract}
Cognitive radio (CR) technology allows secondary users (SUs) to exploit the wireless resources not utilized by licensed primary users (PUs). SU channel selection for finding transmission opportunities incurs non-negligible costs and is a key challenge in successful operation of Cognitive Radio Networks (CRNs). In this paper we present current research studies on algorithms for finding the optimal channels supporting SU Quality of Service (QoS) requirements.
\end{abstract}

\section{Categories and Subject Descriptors}

C.2.1 [Computer-Communication Networks]: Network Architecture and Design - Wireless communication

; F.2 [Analysis of Algorithms and Problem Complexity]: Miscellaneous

\section{Keywords}

Cognitive radio, resource management, channel discovery.

\section{INTRODUCTION}

Cognitive radio (CR) is the technology indicated to provide dynamic spectrum access (DSA) for unlicensed secondary users (SUs) to operate in spectrum bands originally allocated only to primary users (PUs). Cognitive Radio Networks (CRNs) operate by having SUs dynamically access the wireless resources left available by PUs and interrupting transmission when PUs claim them back. An example of coexistence between PUs and SUs is illustrated in Figure 1. Examples of possible applications include IEEE 802.22 Standard, public safety, cellular and mobile ad-hoc networks.

CRNs shall provide support for SU QoS requirements, such as throughput and delay, without harming PUs operating in the same set of channels. Specifically, SUs search for channel opportunities, within a heterogeneous multi-channel $\mathrm{CRN}$, that can guarantee their desired data rate. In case of incoming PU activity, they need to find alternative channels that will sustain their traffic needs. Searching for new

Permission to make digital or hard copies of all or part of this work for personal or classroom use is granted without fee provided that copies are not made or distributed for profit or commercial advantage and that copies bear this notice and the full citation on the first page. To copy otherwise, to republish, to post on servers or to redistribute to lists, requires prior specific permission and/or a fee.

CoNEXT Student'12, December 10, 2012, Nice, France.

Copyright 2012 ACM 978-1-4503-1779-5/12/12 ...\$15.00.

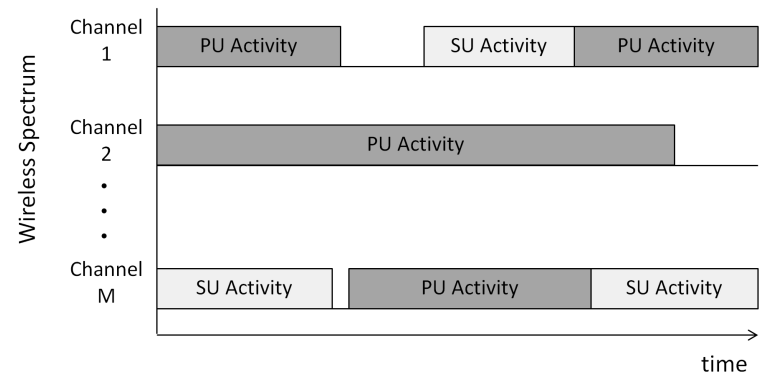

Figure 1: An example of CRN activity.

resources incurs a delay, since it is generally performed sequentially over multiple channels using a single transceiver, by means of spectrum sensing and channel estimation. Conversely, the more channels are searched, the higher the likelihood of finding better resources to support the desired QoS. The trade-off between these two aspects is the target of the channel selection problem, which aims to find the optimal sensing order and optimal stopping rule (i.e., stop or continue sensing channels for finding better and/or more resources) to satisfy SU requirements.

The sensing order and stopping rule are strictly related to each other and depend on CRN characteristics (i.e., number of channels, sustainable data rates, sensing time) and PU activities (i.e., busy/idle probabilities, activity durations, tolerable interference time). We have identified the need to research and develop optimal channel selection algorithms to provide QoS for SUs in heterogeneous CRNs environments.

\section{RELATED WORK}

The problem of channel selection has already been approached in recent works. Authors in $[1,2]$ study the optimal stopping problem for aggregating multiple channels or finding the best channel to use for transmission. Sensing order studies are conducted in $[3,4]$ showing how CRN characteristics affect SU performance differently. To the best of our knowledge, the joint effect of selecting the optimal sensing order and stopping rule have never been studied. Also, current research only considers very limited CRN scenarios.

\section{PROBLEM STATEMENT}

An overlay CRN (i.e., SUs stop transmitting if PUs claim resources back) consisting of $M \mathrm{PU}$ communicating pairs, each operating on a separate non-overlapping channel, is considered. SU pairs opportunistically transmit on the same 


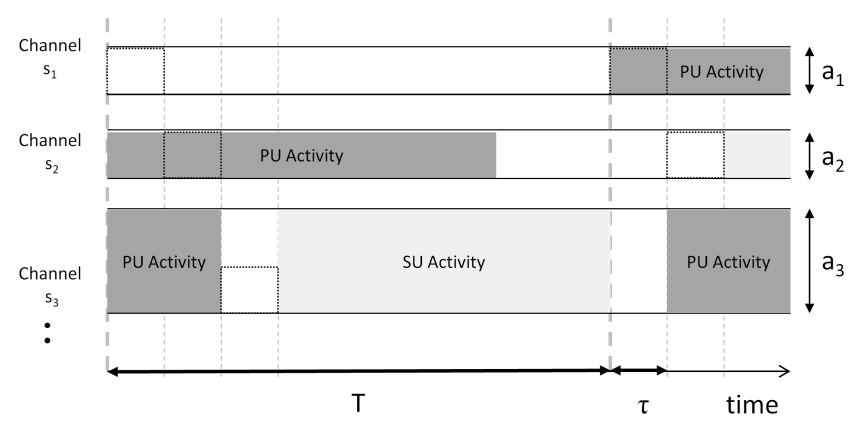

Figure 2: Example of Aggregate Channels Selection.

set of channels, guaranteeing that interfering time to PUs does not exceed a maximum interference tolerance time $T$.

When the SU transmitter node has data to send to its destination, it selects a channel $i(1 \leqslant i \leqslant M)$ and performs channel sensing and channel estimation for a total time $\tau$, identifying possible PU activity and the quality of the wireless channel. If a channel $i$ is found idle, which happens with probabilities $\boldsymbol{Q}=\left[q_{1}, q_{2}, \ldots, q_{M}\right]$, channel estimation reports its maximum achievable data rate $r_{i}$. We model the quality of channel $i$ as a probability distribution $\boldsymbol{P}^{(i)}=\left[p_{1}^{(i)}, p_{2}^{(i)}, \ldots, p_{K}^{(i)}\right]$ of the set of supported maximum data rates $\boldsymbol{R}=\left[r_{1}, r_{2}, \ldots, r_{K}\right]$ with $r_{1}<r_{2}<\ldots<r_{K}$.

Firstly, a channel selection sequence that minimizes the delay for finding the best resources need to be found: $\boldsymbol{S}_{\boldsymbol{M}}=$ $\left\langle s_{1}, s_{2}, \ldots s_{M}\right\rangle$, with $s_{m}$ representing the channel sensed at step $m$. Depending on the CRN characteristics, performance differences between random, decreasing expected data rate or fixed channel orders may vary significantly.

Then, given $S_{M}$, SU needs to make a decision at each time slot $\tau$ if the current achievable throughput (i.e., amount of bits transmitted during $T$ ) can be improved by utilizing another channel $i^{\prime}$ or the additional time lost in sensing and estimating $i^{\prime}$ will cancel the benefit of finding the better resource. This problem can be modeled as a finite-horizon stopping problem as follows. Given the achievable data rate $A(m)$ obtained after estimating $m$ channels, $y_{m}$ represents the current throughput per interval $T$ defined as:

$$
y_{m}=A(m) \max \left\{\frac{T-m \tau}{T}, 0\right\}
$$

find:

$$
\begin{array}{ll}
\min m & \\
\text { subject to } & y_{m} \geqslant \max _{m+1 \leqslant n \leqslant M} y_{n} \\
& 1 \leqslant m \leqslant M
\end{array}
$$

By considering different $A(m)$ formulations, different problems can be analyzed. So far we have identified:

$$
\begin{aligned}
& \text { 1. Single Best Channel: } A(m)=\max _{1 \leqslant j \leqslant m}\left\{a\left(s_{j}\right)\right\} \\
& \text { 2. Aggregate Channels: } A(m)=\sum_{j=1}^{m} a\left(s_{j}\right)
\end{aligned}
$$

with $a\left(s_{j}\right)$ current data rate obtained from channel $s_{j}$. An example of the problem considered for the case of single best channel is shown in Figure 2. An SU communicating pair searches for the maximum throughput attainable during time $T$. It starts sensing from channel $s_{1}$ and finds it idle, but nonetheless it decides to search for a better channel, which is found at the third $\tau$ slot. Channel $s_{3}$ supports a data rate that compensates for the delay occurred.

\section{PRELIMINARY RESULTS}

We already tackled in [5] the problem of finding the single best channel under heterogeneous network conditions and multiple sensing order strategies. By using an approach developed from the optimal stopping rule in [2], we have proposed an algorithm that incorporates the historical statistics of channels and PU activities to find the optimal channel that maximizes SUs throughput. By considering only the combinations with repetitions of possibly attainable data rates, the algorithm has $O(M \cdot K)$ time complexity, which is a major improvement from the typical exponential time of optimal stopping rule solutions. Simulation results show how under most CRN scenarios our scheme produces better channel selections, resulting in throughput improvements as high as $70 \%$. Also, we have evaluated the applicability of channel sensing order strategies under different CRN.

Currently, we are developing an algorithm to reduce the time complexity of the aggregate channels problem. Since all the possible unique aggregate data rate sums need to be considered, the optimal stopping rule with typical backward induction solution would have $O\left(K \cdot M^{K}\right)$ time complexity, which becomes a significant limit when $M$ is large.

\section{FUTURE RESEARCH}

Our research will be extended into two directions. First, we need to generalize the channel selection problem to the case of multiple SU pairs. We foresee the challenge of sharing the dynamically changing $\mathrm{CRN}$ resources among multiple SUs. Sub-optimal channel selection solutions should provide adequate fairness while minimizing performance loss. Secondly, we need to develop and validate statistical models for PU activity and channels quality that can accurately reproduce the future CRN behavior. These statistics would need to be re-evaluated with a frequency dependent on the particular CRN considered.

\section{REFERENCES}

[1] H. T. Cheng and W. Zhuang. Simple Channel Sensing Order in Cognitive Radio Networks. Selected Areas in Communications, IEEE Journal on, 29(4):676 -688, Apr. 2011.

[2] J. Jia, Q. Zhang, and X. Shen. HC-MAC A Hardware-Constrained Cognitive MAC for Efficient Spectrum Management. Selected Areas in Communications, IEEE Journal on, 26(1):106 -117, Jan. 2008.

[3] C.-W. Wang, L.-C. Wang, and F. Adachi. Modeling and Analysis for Reactive-Decision Spectrum Handoff in Cognitive Radio Networks. In GLOBECOM 2010, pages $1-6$, Dec. 2010.

[4] L.-C. Wang, C.-W. Wang, and F. Adachi. Load-Balancing Spectrum Decision for Cognitive Radio Networks. Selected Areas in Communications, IEEE Journal on, 29(4):757 -769, Apr. 2011.

[5] L. Zappaterra, J. Gomes, A. Arora, and H.-A. Choi. A Polynomial-Time Algorithm for Optimizing Channel Sensing Order and Stopping Rule for Heterogeneous Cognitive Radio Networks. Submitted to INFOCOM 2013, Jul. 2012. 